\title{
Internal variability in Arctic regional climate simulations: case study for the SHEBA year
}

\author{
Annette Rinke $^{1, *}$, Philippe Marbaix ${ }^{2}$, Klaus Dethloff ${ }^{1}$ \\ ${ }^{1}$ Alfred Wegener Institute for Polar and Marine Research, Telegrafenberg A43, 14473 Potsdam, Germany \\ ${ }^{2}$ Institut d'Astronomie et de Géophysique G. Lemaître, Université Catholique de Louvain, Chemin du Cyclotron 2, \\ 1348 Louvain-la-Neuve, Belgium
}

\begin{abstract}
The sensitivity of a regional climate model (RCM) to lateral boundary forcing (by different numerical weather prediction analysis products and by various temperature perturbations) and to the initial conditions is evaluated for a pan-Arctic domain. The study focuses on seasonal simulations over the period of the Surface Heat Budget of the Arctic Ocean project from October 1997 to September 1998. Small perturbations in the initial and/or lateral boundary conditions can make the model diverge from driving large-scale fields, and the extent to which this occurs depends on the control of the model by the lateral boundary forcing, not on the origin of the perturbation. The model response to a perturbation does not depend on the type of perturbation, and it is largely independent of the magnitude as well as of the source of the perturbation. Both small and large temperature perturbations have similar consequences for the monthly mean atmospheric patterns and the root mean square difference, but the model response depends on the season. Two regimes in the internal variability were found: (1) large variability in autumn/winter and (2) smaller variability in summer. The pronounced magnitude of internal variability must be taken into account in discussing the significance of climate change and climate sensitivity signals in Arctic RCMs.
\end{abstract}

KEY WORDS: Regional climate modeling · Internal variability · Arctic climate Resale or republication not permitted without written consent of the publisher

\section{INTRODUCTION}

The need for regional climate model (RCM) simulations is increasing in the context of regional integrated assessments and socio-economic applications. Reliable estimates of future climate and its impacts require reliable RCM simulations. For the Arctic domain, work on the quantification of the biases of and scatter between different RCMs has begun recently with the Arctic Regional Climate Model Intercomparison Project (M. Tjernström et al. unpubl., A. Rinke et al. unpubl.; see http://curry.eas.gatech.edu/ARCMIP/index.html).

RCMs constitute complex dynamical systems characterized by substantial nonlinearity. Therefore, each single simulation represents only one realization of possible atmospheric states and contains an implicit uncertainty based on the effects of internal nonlinear processes. RCM simulation uncertainty is caused by uncertainties in initial and boundary conditions, model parameterizations, and numerical algorithms in solving the nonlinear model equations. Internal model variability caused by the nonlinearity of the model system can modulate, or even mask, physically forced signals in RCMs (Giorgi \& Bi 2000, Weisse et al. 2000, Christensen et al. 2001). Therefore, it is essential to know the magnitude of this internal variability to assess the significance of climate change and climate sensitivity signals in RCMs. The aim of the present study was to quantify this magnitude in a pan-Arctic RCM.

Specification of the initial condition (IC) and of the lateral boundary conditions (LBC) is one of the crucial points in RCM construction (e.g. Vukicevic \& Errico 1990, Warner et al. 1997, Denis et al. 2002). Choosing the IC is problematic due to the difficulty of resolving atmospheric structures over data-sparse areas such as the Arctic. The LBC data can be of poor quality as well, due to their coarse temporal and spatial resolutions. They are provided either by global models or numeri- 
cal weather prediction (NWP). Both have resolutions coarser than those used in current RCMs and do not allow parameterizations of specific Arctic processes such as stable boundary layer or aerosol-cloud interactions. The 2 NWP analysis products most widely used are the National Center for Environmental Prediction (NCEP) /National Center for Atmospheric Research (NCAR) analysis, and the European Centre for Medium-Range Weather Forecasts (ECMWF) analysis. Their accuracy is uncertain with regard to the Arctic (see Bromwich et al. 2000, Cullather et al. 2000, Serreze \& Hurst 2000, Francis 2002). Furthermore, the LBC can have a significant impact on the evolution of the predicted atmospheric fields through the propagation of boundary errors into the interior of the domain. LBC can produce transient gravity-inertia modes in the domain. Additionally, an ill-posed mathematical formulation of the LBC can cause spurious interactions between the model solution and the lateral boundaries. Marbaix et al. (2003) present an evaluation of the boundary relaxation methods used in state-of-the-art RCMs.

For more than a decade, a considerable amount of work has been carried out on the skill and uncertainty in regional numerical models in the field of medium and extended range weather forecasting (e.g. Dalcher \& Kalnay 1987, Simmons et al. 1995, Ehrendorfer 1997, Orrell et al. 2001). The experiences with data assimilation and forecast experiments suggest that the largest forecast errors are more likely to arise from errors in the IC than from errors in the model formulation. In this regard, RCM behavior contrasts the behavior of global models. Anthes et al. (1989) found that LBC exert a much stronger control over the error growth than the IC and the use of different model physical parameterizations. These issues have received little attention ever since regional model methodology has been adapted to climate simulations.

The specific feature of an RCM for a large circumpolar Arctic domain is that lateral boundary control is weaker than in mid-latitude and smaller integration domains (Rinke \& Dethloff 2000). Therefore, the panArctic model has more degrees of freedom for deviation from the forcing fields. As Seth \& Giorgi (1998) have argued, this greater freedom facilitates testing of the sensitivity to internal model processes, because the model solution is more free to respond to variations in internal parameters. Nevertheless, the magnitude of the internal variability of the model must be known, to assess the significance of climate sensitivity signals. Few studies have specifically addressed this uncertainty in Arctic or smaller sub-Arctic RCMs (Lynch \& Cullather 2000, Rinke \& Dethloff 2000, Caya \& Biner 2004, Wu et al. 2004).

The objective of this study was to investigate the model's variability, which originates from both IC and
LBC uncertainties under the specific pan-Arctic conditions, and to estimate the effect of internal variability on the simulated climate. If lateral boundary constraints are weaker, i.e. large-scale atmospheric circulation is more strongly decoupled from the driving data, perturbations in the LBC should have a smaller impact. On the other hand, LBC perturbations, as well as any other change between simulations, have an impact on the activity connected with instability processes (e.g. of convective, and in our domain, baroclinic types). Such consequences are associated with the internal model variability, which may be much larger than the perturbation itself. In a pan-Arctic domain, a perturbation is expected to stay within the domain for a longer time. From this point of view, one may expect that LBC perturbations could have a stronger impact.

\section{MODEL AND EXPERIMENTAL SET-UP}

\subsection{Model description}

The model used in this study was the regional atmospheric climate model HIRHAM developed by Christensen et al. (1996) for a pan-Arctic domain (Arctic region north of $65^{\circ} \mathrm{N}$ with $110 \times 100$ grid points, and a grid point spacing of $50 \mathrm{~km}$ ). In addition, a smaller domain with $66 \times 53$ grid points covering the Beaufort and Bering Seas was used in a sensitivity experiment. The model has 19 vertical layers up to $10 \mathrm{hPa}$. The lateral forcing was taken from NWP analysis products and updated every $6 \mathrm{~h}$. The lateral boundary scheme was the simplified Davies (1976) relaxation with a 10 grid point wide boundary zone. HIRHAM contains the physical parameterization package of the general circulation model ECHAM4 (Roeckner et al. 1996), which includes radiation, clouds, convection, as well as planetary boundary, sea surface and land surface processes, and gravity wave drag. A detailed description of the dynamical and physical properties of the model was given by Dethloff et al. (1996).

\subsection{Simulation set-up}

Simulations were conducted for the SHEBA year (Surface Heat Budget of the Arctic Ocean; Uttal et al. 2002) from September 1997 to September 1998. The simulations were performed for 4 mo seasons: September to December 1997 (autumn), December 1997 to March 1998 (winter), March to June 1998 (spring), and June to September 1998 (summer).

For the control simulation ('EC_Ctrl'), the model was initialized and laterally forced by the ECMWF opera- 
Table 1. List of all HIRHAM experiments. LBC: lateral boundary conditions; IC: initial conditions; SST: sea surface temperature

\begin{tabular}{|lll|}
\hline & Expt & Description \\
\hline Control run & EC_Ctrl & LBC forcing and IC from ECMWF analysis \\
LBC and IC effects & NC_Ctrl & LBC forcing and IC from NCEP re-analysis \\
LBC effects & EC_rnd1.0_Expt1 & EC_Ctrl perturbed at LBC by a random perturbation of -1 to $+1 \mathrm{~K}$ \\
& EC_rnd1.0_Expt2 & \\
& EC_rnd0.1 & EC_Ctrl, perturbed at LBC by a random perturbation of -0.1 to $+0.1 \mathrm{~K}$ \\
& NC_rnd1.0_Expt1 & NC_Ctrl perturbed at LBC by a random perturbation of -1 to $+1 \mathrm{~K}$ \\
& NC_rnd1.0_Expt2 & \\
IC effects & EC_clim_rnd1.0 & EC_clim (see below) perturbed as in EC_rnd1.0 \\
EC_Ctrl+1d & As EC_Ctrl, but simulation started 1 d later \\
Lower boundary effects & EC_Ctrl+5d & As EC_Ctrl, but simulation started 5 d later \\
& EC_clim & As EC_Ctrl, but with climatological SST as lower forcing
\end{tabular}

tional analyses (resolution: $2 \times 2^{\circ}, 31$ levels). At the lower boundary, daily ECMWF sea surface temperatures (SSTs) and sea-ice fractions were used. This lower boundary forcing data set was used for all sensitivity experiments (summarized in Table 1), and simulations were performed over all seasons. These sensitivity experiments differ from the control simulation in their LBC and/or IC:

In the first approach, the 2 different NWP analysis products were used as LBC driving data for the simulations. In the first sensitivity experiment, NCEP/NCAR re-analysis data (resolution: $2.5 \times 2.5^{\circ}, 28$ levels) were used for initialization and lateral forcing (instead of ECMWF, as in 'EC_Ctrl'). This experiment is labeled as 'NC_Ctrl'.

The second approach introduced random temperature perturbations at the lateral boundaries; these were constructed in a similar way as in Giorgi \& Bi (2000). The perturbation at a given model grid point is defined as a value within a certain range produced by a random number generator. This perturbation, which randomly changes at each grid point (but only within a buffer area), vertical level, and time step, is added to the temperature field used for the model LBC. The experiments were limited to the temperature changes, because this is one of the key variables in climate change scenario simulations. These perturbation experiments are labeled 'rnd1.0', indicating a perturbation range of -1 to $+1 \mathrm{~K}$, and 'rnd0.1', indicating a perturbation range of -0.1 to $+0.1 \mathrm{~K}$. These perturbation experiments were carried out for both ECMWF and NCEP forcing data (e.g. in the experiment labeled 'EC_rnd1.0', the ECMWF temperature forcing data were perturbed with random values in the range of -1 to $+1 \mathrm{~K})$. The 'rnd1.0' experiment was started twice ('rnd1.0_Expt1' and 'rnd1.0_Expt2'). Note that both experiments have exactly the same experimental setup, but use randomly different perturbations.
The third approach investigated the effects of initial condition changes. For this purpose, the start date was shifted, i.e. the simulation set-up was the same as in the control run ('EC_Ctrl'), except that the simulations were started few days later. These experiments are labeled 'EC_Ctrl+1d' and 'EC_Ctrl+5d', indicating that their start date was shifted by 1 and 5 d, respectively.

\subsection{Evaluation methods}

The deviations between the sensitivity runs and the control run were investigated with the following diagnostics, calculated between pairs of simulations: gridded monthly and seasonal mean differences, spatial root mean square difference (RMSD), and Hovmoeller plots (time-longitude cross-section) at a certain latitude. For the latter, the model variable (e.g. geopotential) was interpolated from the model grid to a chosen geographical latitude and each geographical longitude $\left(0.5^{\circ}\right.$ steps $)$ along this latitude. This was calculated in a loop over the model write-out time $(6 \mathrm{~h})$.

These calculations are presented for the model variable 'temperature of the lowest model level' (in the following termed $\mathrm{T}_{1}$ ). The lowest model level is at a height of about $30 \mathrm{~m}$ above ground. As we inspected other model fields, e.g. the geopotential height and mean sea level (MSL) pressure, the results presented here are representative of the model's behavior.

\section{RESULTS}

\subsection{Implications of the use of ECMWF or NCEP analysis data as lateral boundary conditions}

The aim in using NWP analysis products as lateral forcing is to have 'perfect' LBCs and to evaluate the 
performance of RCMs. Evaluation of the different NWP analysis products from NCEP and ECMWF (see 'Introduction') shows that each (re)-analysis has its own strengths and weaknesses in different fields, and that they largely differ in the Arctic.

\subsubsection{Differences between NCEP and ECMWF data}

Fig. 1 shows a representative example of the differences between the ECMWF operational analyses and the NCEP re-analyses for autumn (seasonal mean difference of NCEP - ECMWF of $T_{1}$ for autumn). Differences range from -6 to $+8 \mathrm{~K}$. The largest differences occur over the Arctic Ocean and along the land-sea and sea-ice borders. For the RCM simulation, only the values at the model's boundaries are used to drive the model. We evaluated these $6 \mathrm{~h}$ instantaneous $\mathrm{T}_{1}$ differences between the NCEP and ECMWF data at the HIRHAM boundary grid points (data not shown). The smallest perturbations are introduced into the RCM along the ocean parts; both analyses agree in this case up to a difference of $1.5 \mathrm{~K}$. The differences over land are up to $4.5 \mathrm{~K}$ and show pronounced temporal changes, with the largest differences in November and December. This reflects the influence of the different land-surface schemes used in the 2 analysis approaches. The temporal evolution of the RMSD between the 2 data analyses is shown in Fig. 2 (light blue dashed curve). The RMSD is between 2 and $6 \mathrm{~K}$.

\subsubsection{Model response to NCEP and ECMWF data}

The response of the model to differences between the data sets was measured by the RMSD (internal variability) and investigated by comparing the 'EC_Ctrl' and 'NC_Ctrl' simulations (Fig. 2). Within the first few days, the RMSD of temperature increased for a spin-up period of 15 to $20 \mathrm{~d}$ in spring and summer, or $10 \mathrm{~d}$ in winter and autumn. After this, the values oscillated around dynamical equilibria with values much higher than the IC. A high RMSD appeared in autumn and winter (equilibrium value of 5 and $6 \mathrm{~K}$, respectively), then it decreased abruptly in mid-spring and reached the smallest values in summer (equilibrium value of $3 \mathrm{~K}$ ), indicating the existence of 2 regimes of internal variability. The temporal classification of the 2 regimes (high variability in autumn/winter; low in spring/summer) is completely inverse to those found by Giorgi \& Bi (2000), Christensen et al. (2001), and Caya \& Biner (2004), who detected the largest internal variability in their RCMs during summer. To understand this different model behavior, it is necessary to sort out the factors which add a strong element of ran-

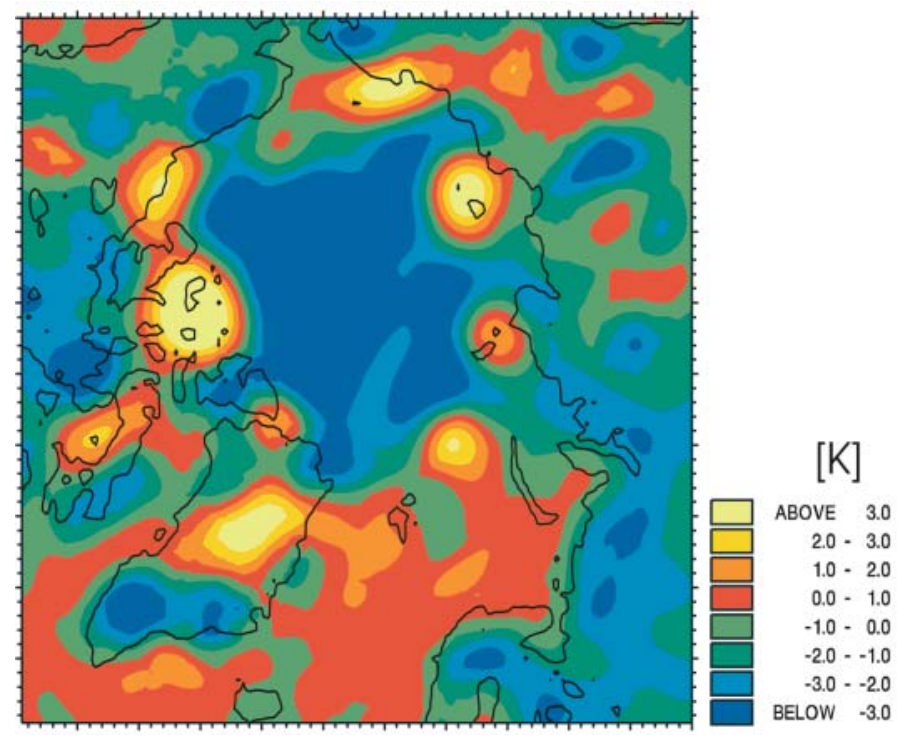

Fig. 1. Seasonal mean differences in the lowest model level temperature $\left(\mathrm{T}_{1}\right)$ between NCEP re-analysis and ECMWF operational analysis in autumn

domness and nonlinearity and therefore maximize the internal model variability. The model domain of Giorgi \& Bi (2000) covers eastern Asia, where thermal instability and moist convective activity are at a maximum during the summer season; during the winter season, when East Asia experiences its dry season, synoptic scale systems dominate the weather of the region, and stronger westerly flows sweep away inter-

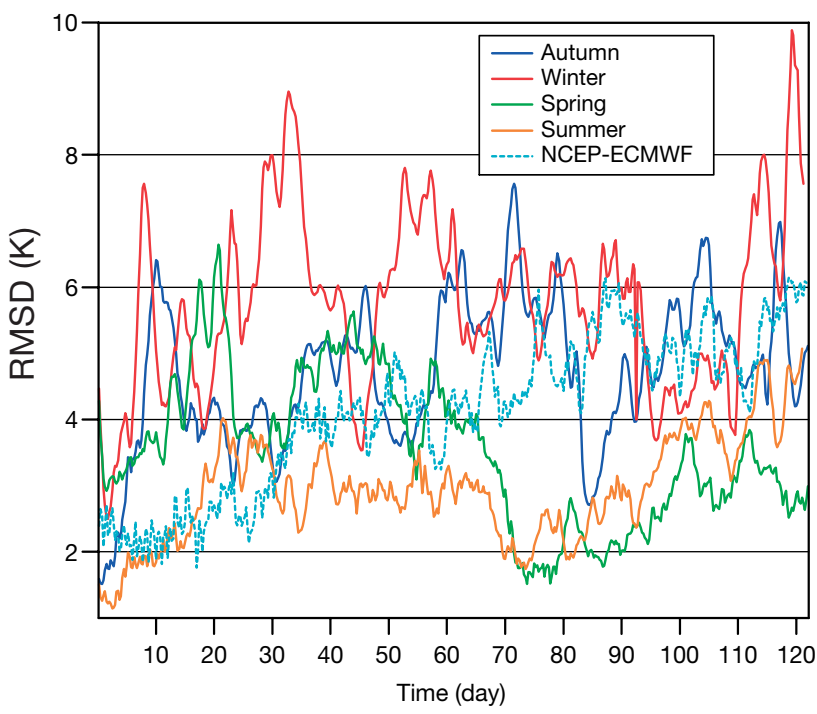

Fig. 2. Root mean square difference (RMSD) between NCEP and ECMWF forced runs ('NC_Ctrl' vs 'EC_Ctrl') for $6 \mathrm{~h}$ temperature at the lowest model level $\left(\mathrm{T}_{1}\right)$ as a function of time by seasons. Dotted: RMSD between driving analyses data 'NCEP' - 'ECMWF' in autumn 
nally generated model responses. The model domains of Caya \& Biner (2004) and Christensen et al. (2001) cover the mid-latitudes, where the prevailing strong zonal circulation mitigates model-generated errors or perturbation responses by a fast propagation out of the model domain, while during the summer there is a weaker zonal circulation and the importance of local processes (e.g. convection) becomes stronger. The circumpolar domain in this study is characterized by dynamical features, which cause a different seasonal regime of variability (see Section 3.4).

In addition, significant variability in the RMSD at scales of a few to $10 \mathrm{~d}$ is superimposed on the seasonal cycle (Fig. 2). This is related to synoptic-scale variability manifested by a correlation of the RMSD with the interdiurnal variability of the MSL pressure (data not shown).

Autumn

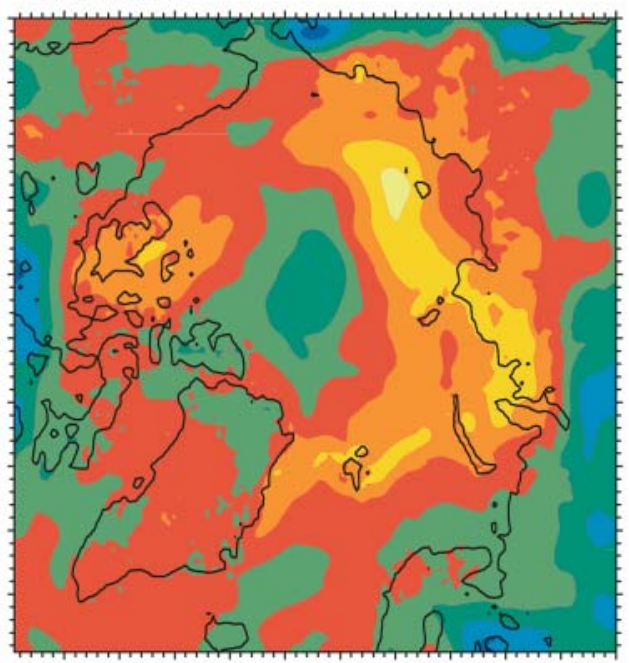

Spring

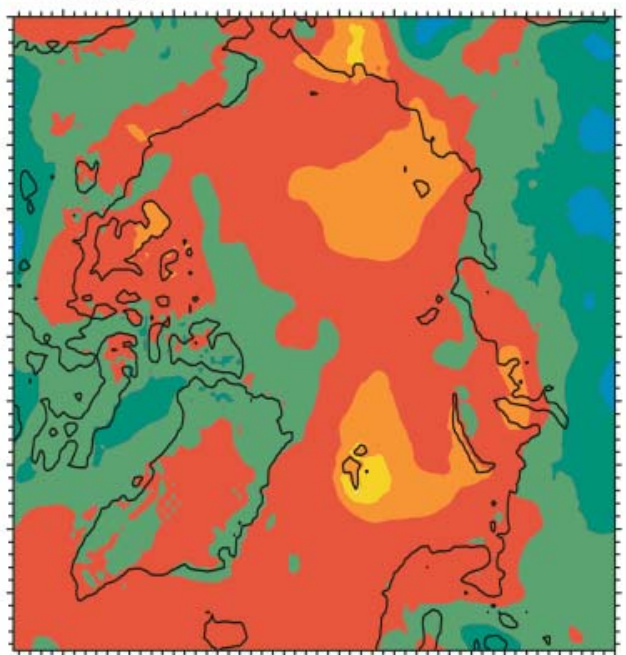

Fig. 3 shows the seasonal mean spatial patterns of the $\mathrm{T}_{1}$ difference between the 2 HIRHAM simulations using either NCEP or ECMWF as the lateral boundary forcing ('NC_Ctrl' - 'EC_Ctrl'). Compared to Fig. 1, the RCM seasonal mean response to the LBC forcing is of the same order, but often larger in magnitude and in spatial scale than the LBC differences themselves. This means that the lateral disturbances are transferred into the interior of the RCM domain and become amplified along the way. The acting mechanisms are the impact of the LBC perturbation on the model-generated circulation and the interaction of the large-scale circulation with the smaller-scale waves within the domain. Fig. 3 shows that the seasonal mean response is smallest in summer and largest in winter. This is a consequence of 2 factors: (1) differences between NCEP and ECMWF are much smaller during summer than in winter,

\section{Winter}

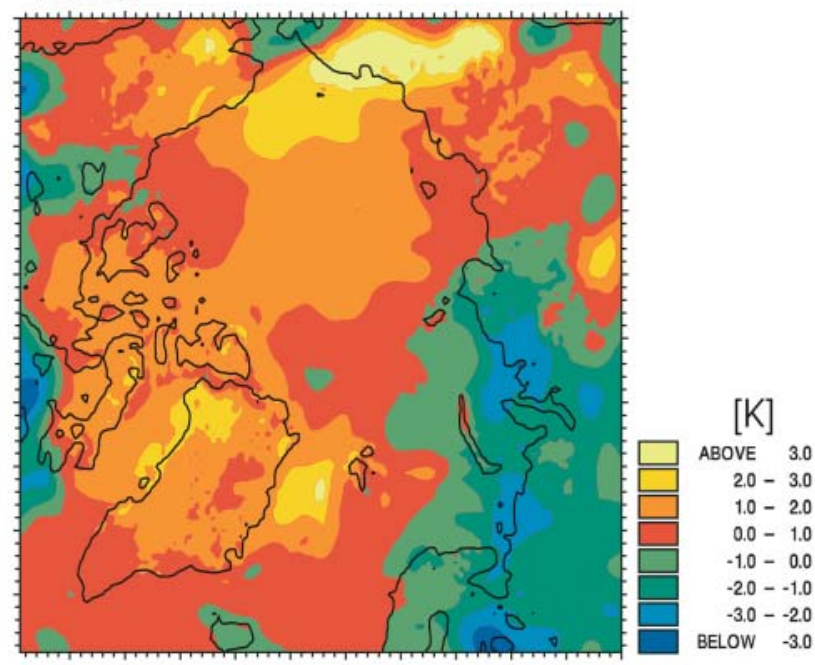

\section{Summer}

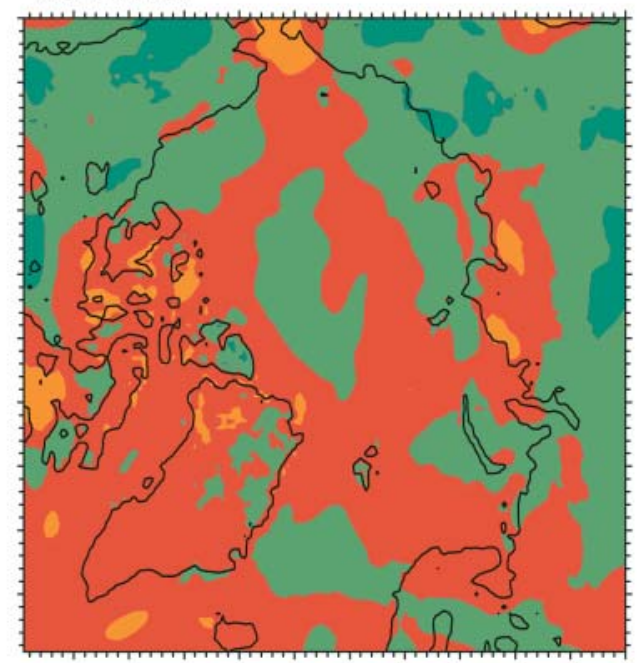

Fig. 3. Seasonal mean difference patterns between the NCEP and ECMWF forced runs 'NC_Ctrl' - 'EC_Ctrl' for temperature at the lowest model level $\left(\mathrm{T}_{1}\right)$ 

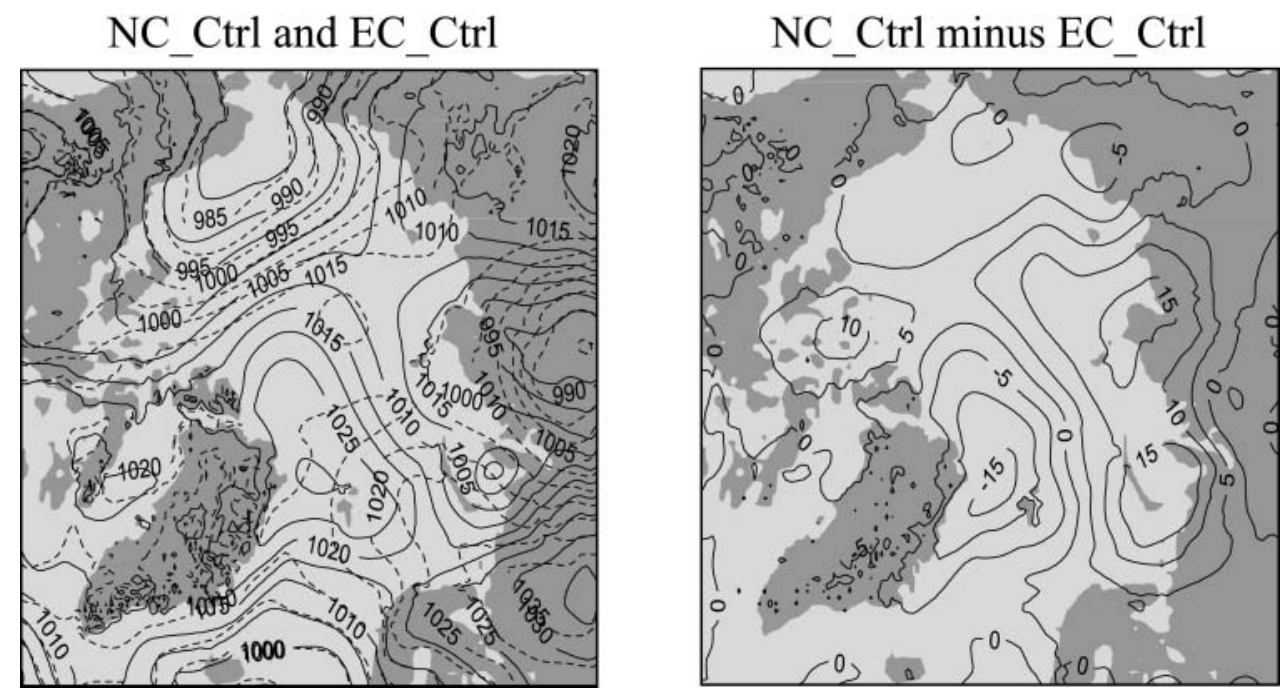

Fig. 4. Mean sea level (MSL) pressure patterns (hPa) for 'NC_Ctrl' (dashed lines) and 'EC_Ctrl' (solid lines) and the difference between both simulations on 11 November at 12:00 h UTC

(2) specific dynamic features of the Arctic circulation cause this seasonal response in the model (see Section 3.4). The response is to some extent fixed at certain geographical regions (East of Greenland-Spitsbergen, and Laptev Sea-eastern Siberia). As an example, Fig. 4 shows the different circulation patterns on the day at which the RMSD reaches its maximum (11 November at 12:00 h UTC; see Fig. 2).

With regard to the synoptic situations, the 'EC_Ctrl' simulation develops a high-pressure system NE of Greenland and a low-pressure system in the Kara Sea,

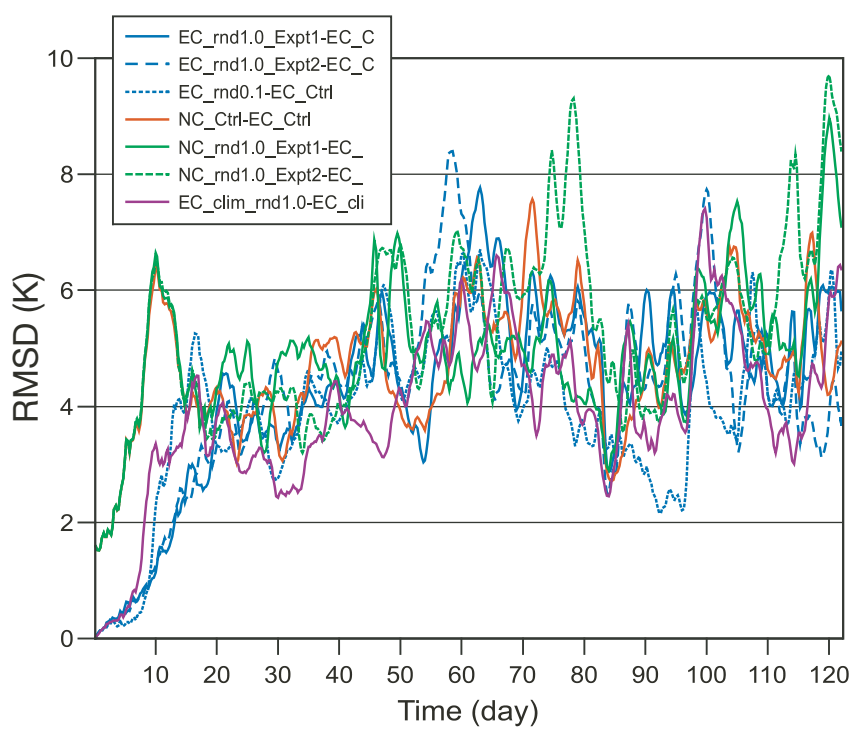

Fig. 5. Root mean square difference (RMSD) between each perturbation experiment listed in Table 1 and the control run 'EC_Ctrl' for $6 \mathrm{~h}$ temperature at the lowest model level $\left(\mathrm{T}_{1}\right)$ as a function of time for autumn while 'NC_Ctrl' tends to develop a ridge over these areas. Three regions may be considered as main contributors to the RMSD: the Fram Strait area, the Canadian Archipelago, and the Barents/Kara Seas.

\subsection{Implications of random perturbations in lateral boundary conditions}

\subsubsection{Temperature}

The temporal evolution of the RMSD between the different random temperature perturbation experiments and the control simulation ('EC_Ctrl') is illustrated in Fig. 5. The most striking feature is the similar evolution of the RMSD for all perturbation experiments. Different perturbation amplitudes led to very similar RMSD amplitudes and temporal behavior. For instance, a small perturbation (Expt 'EC_rnd0.1') caused a similar response amplitude as a large perturbation Expt 'EC_rnd1.0'). Thus, the amplitude of the perturbation at the lateral boundaries does not influence the simulated perturbation response. In addition, the simulated response does not depend on the type of perturbation. It can be concluded that the model's RMSD response to a temperature perturbation is largely independent of the magnitude as well as of the type or source of perturbation.

\subsubsection{Pressure}

The pressure patterns of the 2 simulations 'EC_rnd1.0' and 'EC_Ctrl' associated with the maxi- 

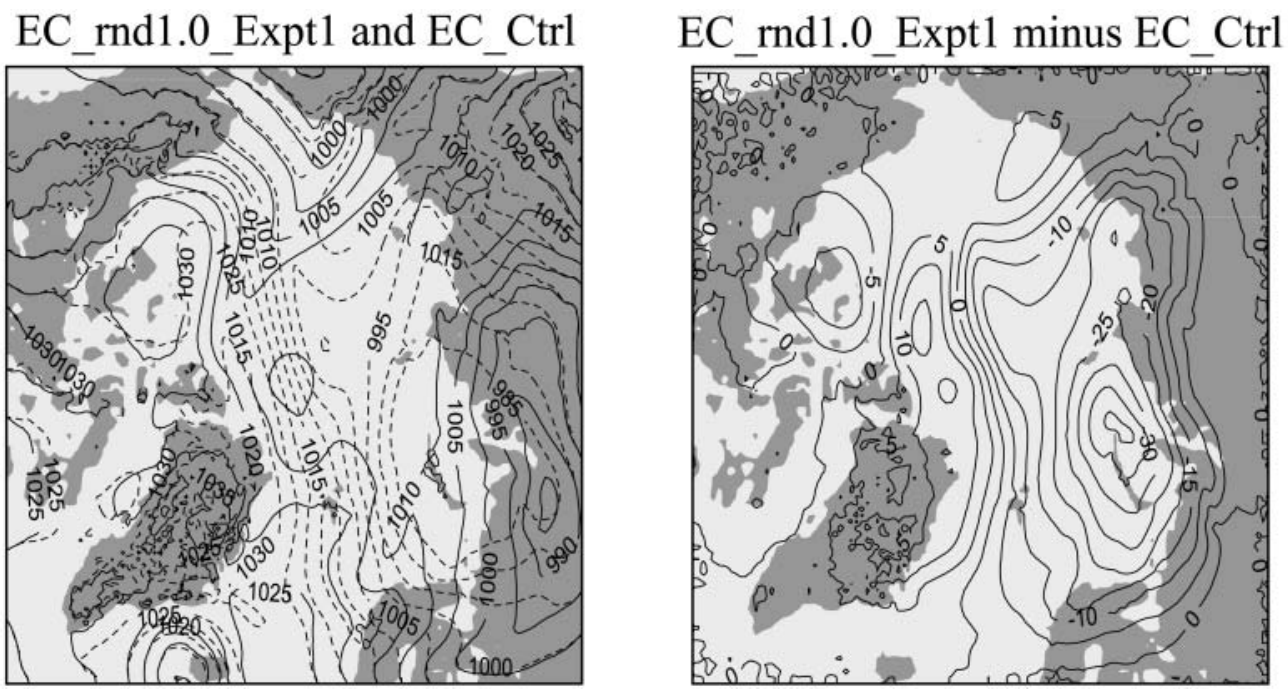

Fig. 6. Mean sea level (MSL) pressure patterns (hPa) for 'EC_rnd1.0' (dashed lines) and 'EC_Ctrl' (solid lines) and the difference between both simulations on 3 November at 00 UTC

mum RMSD (on 3 November at 00:00 h UTC) show that the synoptic situations for both simulations are completely different (Fig. 6). The main contributors to the RMSD of MSL pressure are the same regions as before: the Canadian Archipelago, and the Barents, Kara, and Laptev Seas (cf. Fig. 4).

\subsubsection{Seasonality}

The RMSD depends again on the season (e.g. Fig. 7 for Expts 'EC_rnd1.0' and 'EC_Ctrl'). Two regimes are found: high internal variability in autumn/winter and low internal variability in spring/summer. The seasonal behavior of the RMSD is similar for the experiments, independent of the type of perturbation (cf. Figs. 2 \& 7). RMSD temporal variation is primarily guided by seasonal variability at scales of a few to several days (Fig. 7). The RMSD in this study is much higher (up to 5 times) than in Caya \& Biner (2004) and Christensen et al. (2001 (see Section 3.4).

The spatial patterns of the model response to the random LBC perturbations are illustrated in Fig. 8 (seasonal mean $\mathrm{T}_{1}$ differences between the HIRHAM simulations 'EC_rnd1.0' and 'EC_Ctrl'). Although the LBC perturbation amplitude is in the range of -1 to $+1 \mathrm{~K}$, there is a $\pm 2 \mathrm{~K}$ perturbation response over most of the domain, with maxima of up to $\pm 4 \mathrm{~K}$ at a few grid points. The conclusion is that the LBC perturbation propagates into the interior of the model domain and becomes modified/amplified there. In the pan-Arctic domain, the large-scale circulation is influenced most strongly by its component around the North Pole, and the perturbation thus remains within the domain because its migration out of the domain is slowed (see Section 3.4).

\subsubsection{Domain effects}

The influence of the integration domain on the calculated internal variability was investigated by comparing the results from the pan-Arctic domain with those from a smaller western Arctic domain. For this purpose, the 'EC_Ctrl' control and the 'EC_rnd1.0' LBC perturbation experiments were repeated for a $66 \times 53$

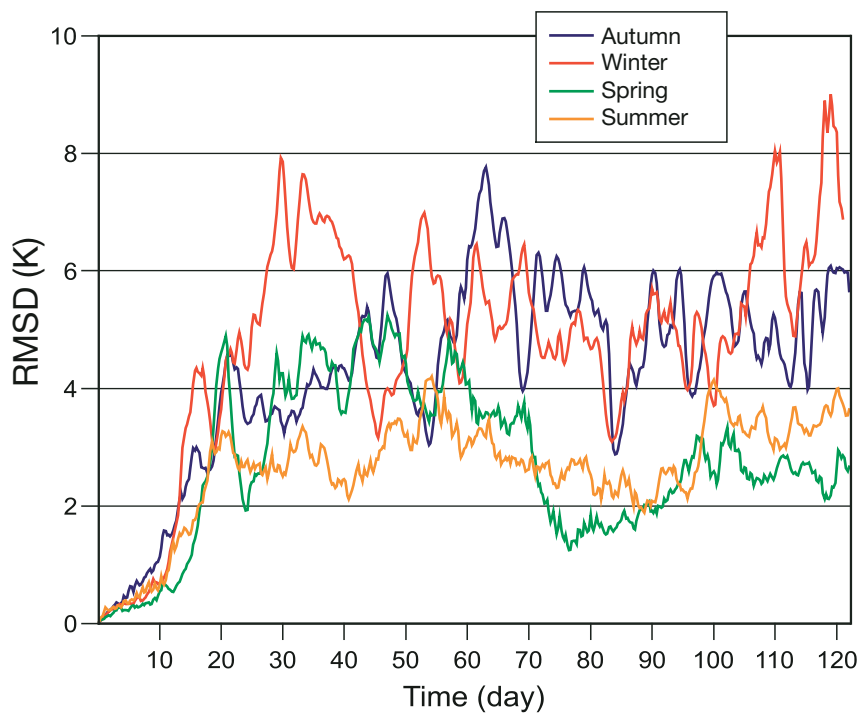

Fig. 7. Root mean square difference (RMSD) between 'EC_rnd1.0' and 'EC_Ctrl' for $6 \mathrm{~h}$ temperature at the lowest model level $\left(\mathrm{T}_{1}\right)$ as a function of time by seasons 

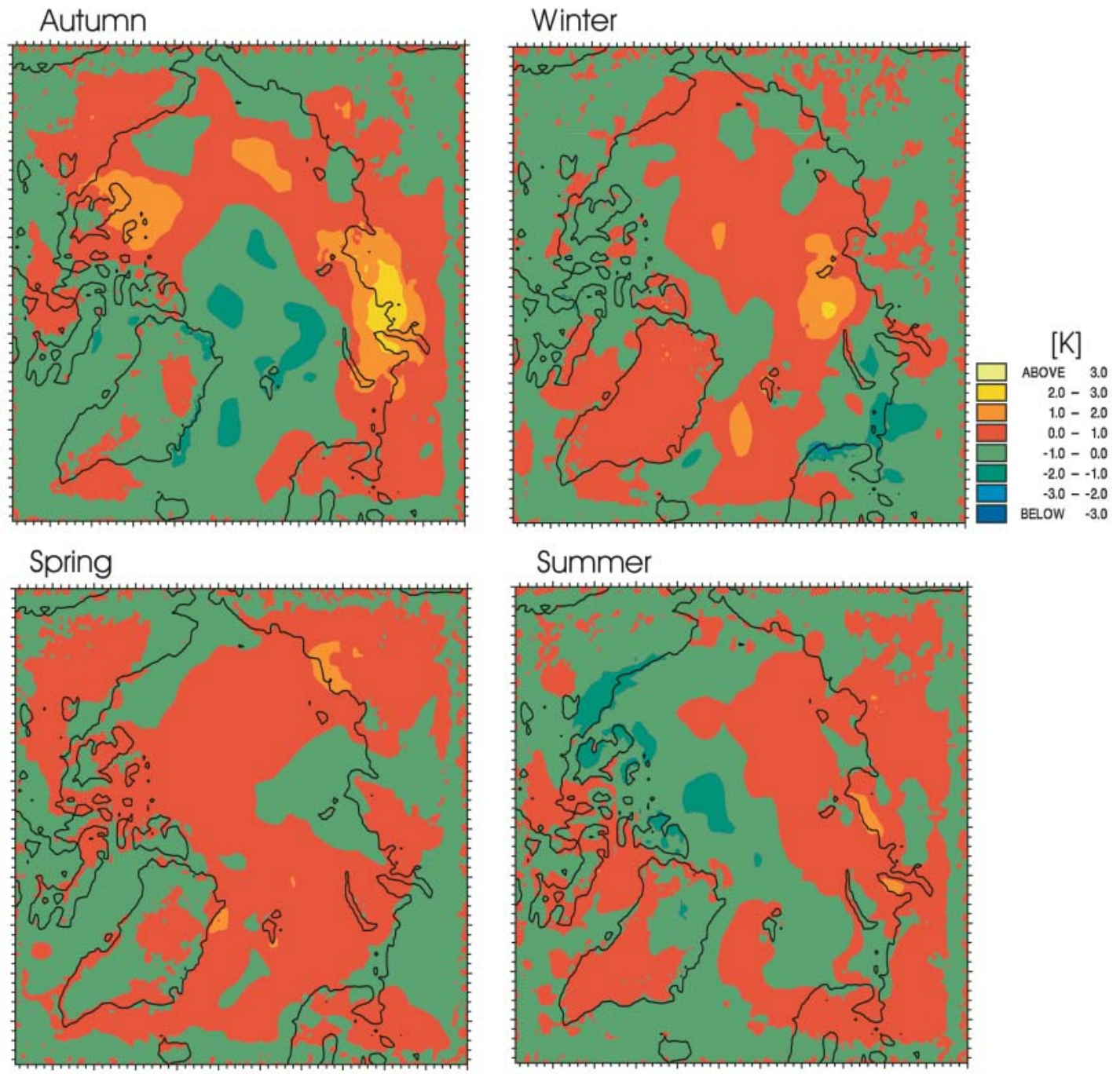

Fig. 8. Seasonal mean difference patterns of 'EC_rnd1.0' - 'EC_Ctrl' for temperature at the lowest model level ( $\left.\mathrm{T}_{1}\right)$

grid point domain for autumn. This domain covers the SHEBA experiment area, i.e. the Beaufort and Bering Seas, and is about a third as large as the pan-Arctic domain (A. Rinke et al. unpubl.; http://cires.colorado. edu/lynch/arcmip/Domain_info.html). Regarding the seasonal mean spatial pattern of the model response caused by LBC temperature perturbation (data not shown), the magnitude of the model response is reduced to the range of -1 to $+1 \mathrm{~K}$ (about one-half to one-fourth smaller than the pan-Arctic domain results). The temporal evolution shows that RMSD is about $0.5 \mathrm{~K}$ (Fig. 9), compared to $5 \mathrm{~K}$ in the pan-Arctic domain (blue curve in Fig. 7). Additionally, there is a large peak in the RMSD around the 70th day (between 7 and 9 November). These days are characterized by deep low pressure systems in the Bering Sea and a strong cyclonic flow from the Bering Strait to the Chukchi Sea connected with strong northward warm air advection. This circulation pattern is affected by the imposed LBC perturbation, indicating that the formation of such meteorological situations reacts very sensitively to perturbations of the LBC and is therefore less predictable than other circulation patterns. The same appears to apply to the pan-Arctic domain (Figs. $2 \& 4$ and Figs. 5 \& 6), especially in autumn and winter.

\subsubsection{Sea surface temperatures and sea-ice}

Mesoscale processes add elements of nonlinearity and complexity to the model, i.e. the strength of these processes particularly influences the amplitude of the internal model variability. During spring and summer, convection and thermal instability over the open ocean and along the sea-ice edges may play an important role in this variability. Therefore, one hypothesis is that the lower boundary forcing (SST, sea-ice distribution) may exert an influence on the internal variability. To 


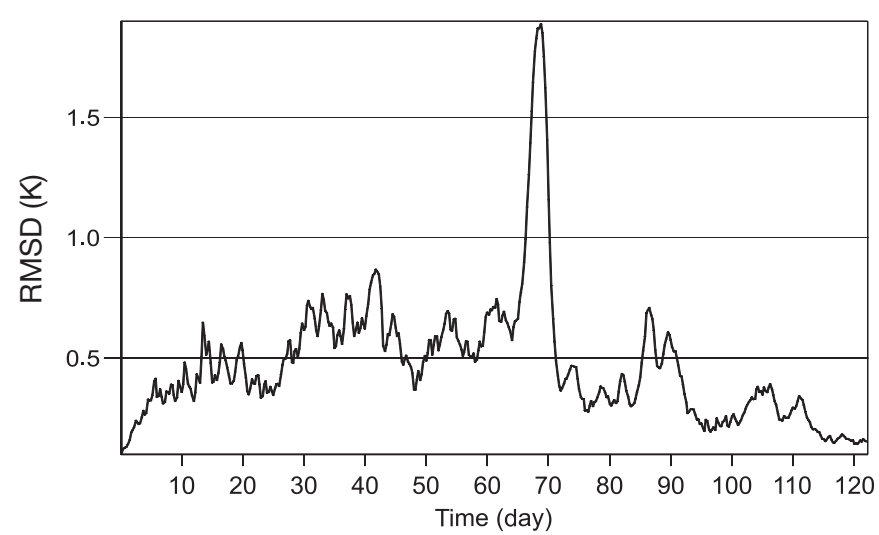

Fig. 9. Root mean square difference (RMSD) between 'EC_rnd1.0' and 'EC_Ctrl' for $6 \mathrm{~h}$ temperature at the lowest model level $\left(T_{1}\right)$ as a function of time in autumn for the small western Arctic model domain

investigate this hypothesis, the control run was repeated using climatological SSTs and sea-ice distribution at the lower boundary (based on Brankovic \& van Maanen 1985). This experiment is labeled as 'EC_clim'. The climatological sea-ice border extends much further southward (100 to $500 \mathrm{~km}$, depending on the region), leading to lower SSTs (up to $3 \mathrm{~K}$, maximum in the Chukchi Sea and SE of Greenland) compared to the year 1997-1998. The climatological sea-ice extent is about 2 million $\mathrm{km}^{2}$ larger in autumn compared to the year 1997-1998 (taken a minimum ice concentration of $15 \%$ to define sea-ice extent). As discussed by Rinke \& Dethloff (2000), such differences in SSTs and sea-ice distribution can modify the atmospheric circulation. Here we discuss the influence of this different lower boundary forcing on the model response to LBC perturbations. For this purpose, a random lateral perturbation experiment was carried out on the 'EC_clim' simulation, labeled as 'EC_clim_rnd1.0'. The calculated perturbation response (purple curve in Fig. 5) did not differ from those of the earlier perturbation experiments. The conclusion is that the LBC perturbation acts independently of SST and ice conditions. There is practically no dependence on local surface forcing: even a strong surface forcing within the domain does not reduce the dominance of the relatively strong sensitivity to LBC perturbations.

\subsection{Implications of different initial conditions}

Rinke \& Dethloff (2000) investigated the pan-Arctic model sensitivity to IC. They found a pronounced sensitivity due to the relatively weak lateral boundary control. In agreement with Giorgi \& Bi (2000), we expected that the effects of perturbations in IC and
LBC could be distinguished from each other, since IC perturbations were applied only once at the beginning of the simulation, while LBC perturbations were applied continuously over time. This assumption was examined by 2 simulations with different IC. The simulation's set-up was identical to the control run ('EC_Ctrl') except that they were started $1 \mathrm{~d}$ ('EC_Ctrl+1d') or $5 \mathrm{~d}$ ('EC_Ctrl+5d') later. The control run and the 2 IC-perturbed runs thus had different atmospheric and surface IC. Fig. 10 shows that the model responses to different initial conditions were very similar to the response to LBC perturbations: the IC-perturbed model response has both the same amplitude and the same temporal evolution as the LBC-perturbed model response.

Our experimental set-up with overlapping seasons permitted the investigation of another aspect of the IC dependence: model response after 4 mo can be compared to the response after 1 mo. For example, the last month of the autumn season (September to December 1997) can be compared with the first month of the winter season (December 1997 to March 1998), etc. Analyzing the RMSD (Figs. $2 \& 7$ ) again under this aspect shows a pronounced difference for the same month. Fig. 11 shows the spatial patterns of the monthly mean $\mathrm{T}_{1}$ differences between the HIRHAM simulations 'EC_rnd1.0' and 'EC_Ctrl' for 2 examples. In the case of December 1997, the magnitude of the model response to the LBC perturbation is very similar, but the spatial patterns of the response are very different. In the case of June 1998, both the magnitudes as well as the patterns of the response differ. These differences are in principle only a result of the different

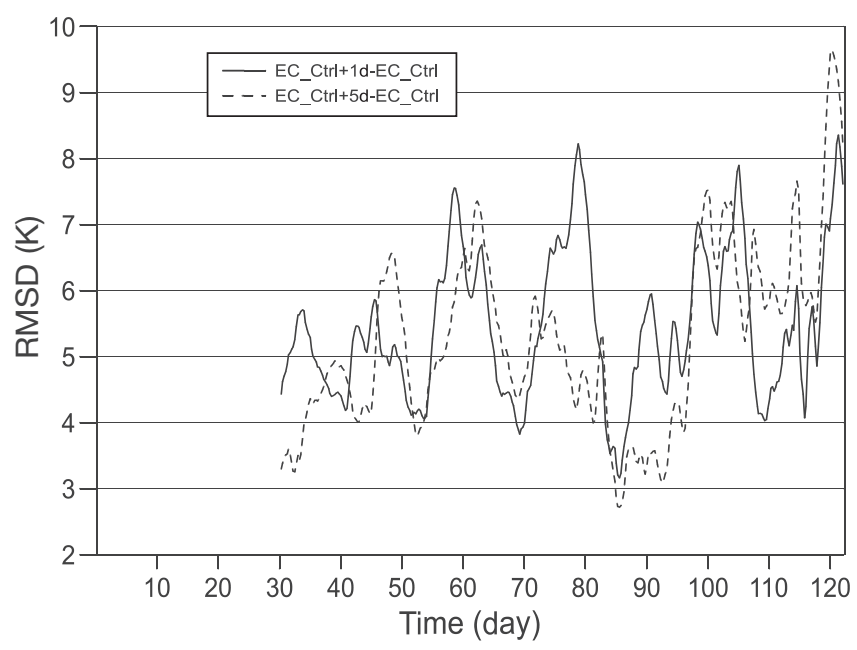

Fig. 10. Root mean square difference (RMSD) between the initial condition perturbation experiments listed in Table 1 and the control run 'EC_Ctrl' for $6 \mathrm{~h}$ temperature at the lowest model level $\left(\mathrm{T}_{1}\right)$ as a function of time in autumn 


\section{(a) December 1997}

\section{Simulation started on 09/01/97}

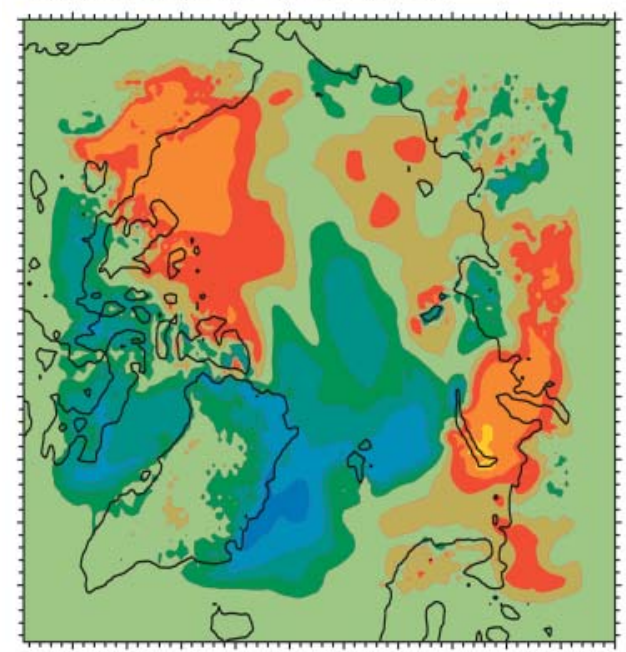

(b) June 1998

Simulation started on $03 / 01 / 98$

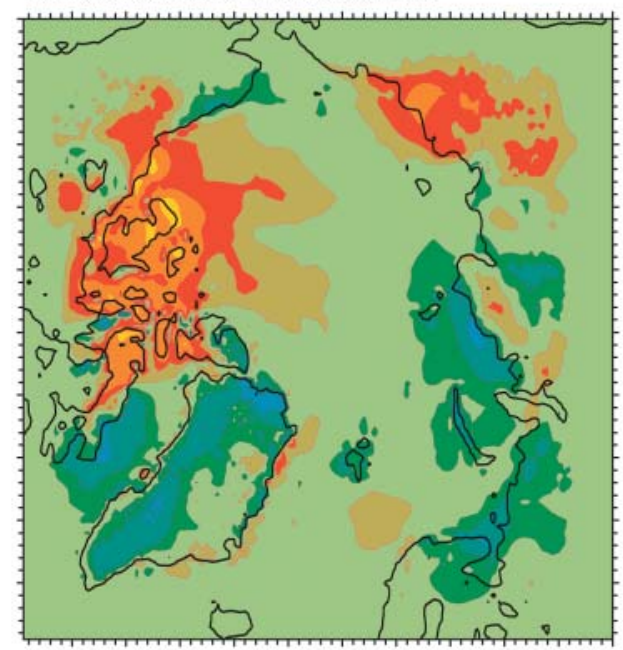

Simulation started on 12/01/97

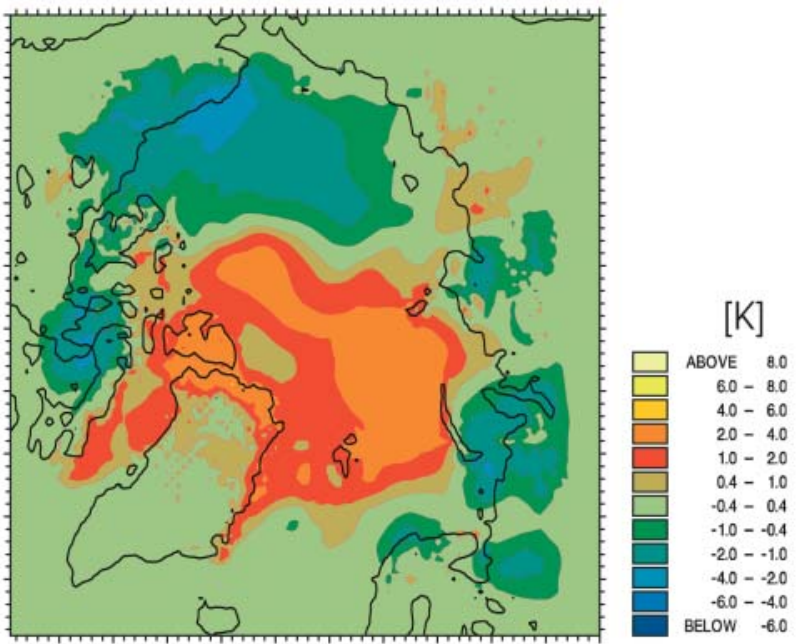

Simulation started on $06 / 01 / 98$

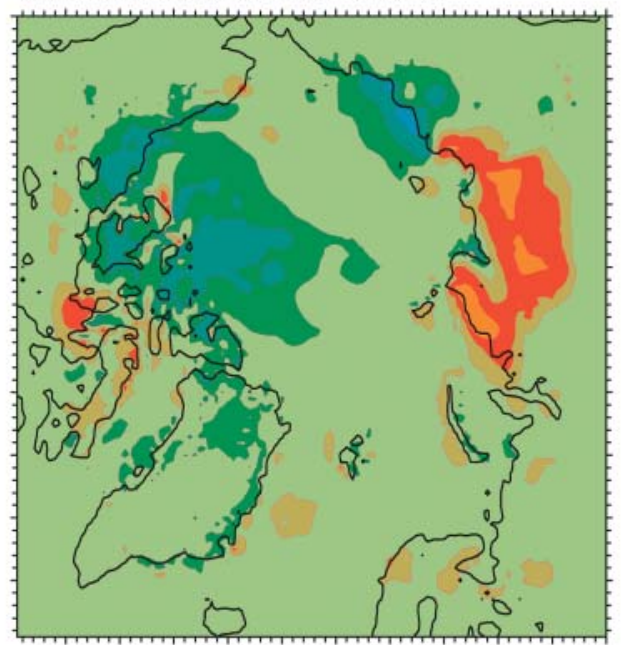

Fig. 11. Monthly mean difference patterns of 'EC_rnd1.0' - 'EC_Ctrl' for temperature at the lowest model level ( $\left.\mathrm{T}_{1}\right)$ after various simulation periods. Dates given as $\mathrm{mo} / \mathrm{d} / \mathrm{yr}$

model initialization (i.e. different start time and duration) and this causes a different response to the same perturbation.

\subsection{Characteristics of the pan-Arctic domain}

The large internal variability and its seasonal regime are unique dynamic characteristics of the pan-Arctic domain. The magnitude is larger and the seasonal regime is completely converse to the results of Giorgi \& Bi (2000), Christensen et al. (2001), and Caya \& Biner (2004).

An imposed perturbation remains within the circumpolar domain for longer time periods than in mid- latitude domains, and therefore the interactions of the perturbations with long planetary waves are much more effective (Rinke \& Dethloff 2000). Arctic circulation is weaker during summer: planetary wave activity is weaker, MSL pressure is characterized by weaker gradients, cyclonic activity is distributed more uniformly, pressure systems tend to be considerably weaker (e.g. Serreze et al. 1993). Especially in winter and autumn, large-scale Arctic circulation is dominated by a strong zonal symmetric (vortex) component, and an imposed perturbation can stay within the domain, because its rapid migration out of the domain is impeded. This specific feature of the pan-Arctic domain is illustrated in a Hovmoeller plot of the $500 \mathrm{hPa}$ geo- 
potential height model response (i.e. 'EC_rnd1.0' 'EC_Ctrl') in autumn and summer Fig. 12. It shows the temporal course of the model response to an LBC perturbation at a fixed geographical latitude $\left(75^{\circ} \mathrm{N}\right)$ along the longitudes. In autumn, a pronounced wave-like pattern (west- and eastward propagation of the response around the $75^{\circ} \mathrm{N}$ circle) can be observed, whereas this wave activity is very small during summer. The LBC perturbation causes a wavelike response of the geopotential in the interior of the model domain, and this interacts with the model generated large-scale and baroclinic waves, whereby the baroclinic instability (which is larger in winter than in summer) may also contribute to the amplified model response. This explains the amplified model response to the LBC and/or IC perturbations and the 2 seasonal regimes found in the RMSD.

\section{SUMMARY AND CONCLUSIONS}

Uncertainties in LBC and IC generate strong internal model variability within a pan-Arctic RCM. The magnitude of this variability, measured by the monthly and seasonal mean spatial pattern differences and the RMSD, is much larger than those of smaller Arctic domains or mid-latitude domains of the same size. This is a special feature of the panArctic domain with its specific dynamical character. The model has a relatively large freedom to diverge from the LBC forcing, and due to its nonlinearity and complexity, it can diverge from the LBC in various ways. In other words, LBC forcing does not limit the effect of uncertainty. Thus, the origin of this uncertainty has no impact on the magnitude of the model response. This means that small changes in IC and LBC can result in a model divergence from the driving large-scale fields. The extent to which this occurs depends on the control of the model by the LBC forcing, not on the origin of the perturbation. The model response to a perturbation is largely independent of the magnitude as well as of the type or origin of the perturbation. LBC and IC perturbations, either of smaller or larger amplitude, have similar consequences on the monthly and seasonal response patterns and the evolution of the RMSD. However, some key geographical regions which contribute most to the RMSD have been identified. These locations may be connected with orographic features and/or sea-ice distribution, which may trigger the spatial model response pattern, but this hypothesis needs to be investigated further.

Two regimes in the internal variability are found: a regime of large variability in autumn/winter and a regime of smaller variability in summer. Atmos-
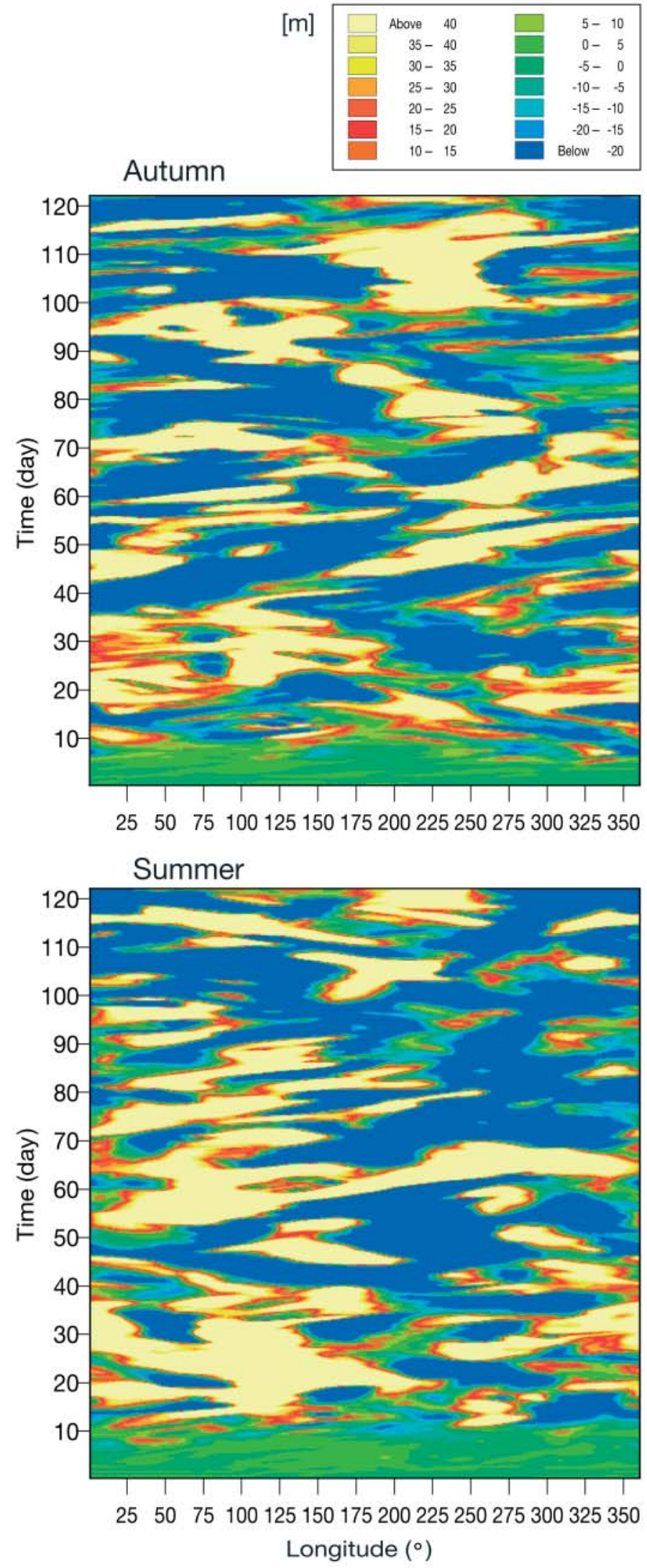

Fig. 12. Hovmoeller plots of the $500 \mathrm{hPa}$ geopotential height difference 'EC_rnd1.0' - 'EC_Ctrl' at $75^{\circ} \mathrm{N}$ latitude for autumn and summer 
pheric circulation and planetary wave activity are weaker during summer than during winter. Therefore, the response of the model to LBC and/or IC perturbations develops (amplifies) at a slower rate in summer, due to the reduced model-generated wave activity and instability. In addition, a changed surface forcing within the domain (e.g. different SST and sea-ice distribution, and thereby changed local heat exchange) does not reduce the dominance of the LBC sensitivity. Models such as the pan-Arctic domain RCM tend to have a large internal variability, triggered by uncertainties in IC and/or LBC. Complementary work with the 'poor-man' nudging procedure (cf. von Storch et al. 2000) and with the more advanced Arctic System reanalysis project (SEARCH 2003) attempt to circumvent these problems by adding a constraint, although this does not seem suitable for investigating the nonlinear interaction and feedbacks within the model system.

The present results have implications for the downscaling of general circulation models (GCMs) in the Arctic. In addition to the large inter-GCM scatter and the large GCM performance biases in the Arctic (Walsh et al. 2002, Räisänen 2002, Covey et al. 2003), 3 findings make Arctic GCM downscaling even more complex: (1) The solutions of different Arctic RCMs scatter considerably, even in a very constrained model set-up, due to their different physics and/or dynamics (M. Tjernström et al. unpubl., A. Rinke et al. unpubl.); this intermodel scatter highlights the magnitude and seasonal dependence of the disagreement and unreliability in current RCM simulations. (2) Each individual RCM has its own large internal variability patterns; large internal variability requires ensemble runs for each individual model to be able to assess significant simulation responses on, e.g., physical parameterization changes. (3) Internal climate fluctuations associated with nonlinear instability and feedback processes are important mechanisms for natural climate changes and compete with external changes (e.g. Dorn et al. 2003, Dethloff et al. 2004). Thus, to obtain more credible estimates of future Arctic climate via GCM downscaling, the use of a multi-GCM forcing and/or a multiRCM model ensemble approach is highly recommended. This means that each different RCM has to perform ensemble simulations (e.g. using different IC and LBC). Two projects with this target are now under way: the GLIMPSE project (www.awi-potsdam.de/ www-pot/atmo/glimpse/ ) and the ARCMIP project).

Acknowledgements. Part of this study was done while P.M. visited the Alfred Wegener Institute, Germany. We thank R. Reeder, who made the NCEP/NCAR re-analyses available to us. We are grateful to I. Hebestadt and S. Erxleben for preparing the graphics and the runs. A.R. and K.D. were supported by the European Union project GLIMPSE (EVK2-CT-2002-00164).

\section{LITERATURE CITED}

Anthes RA, Kuo YH, Hsie EY, Low-Nam S, Bettge TW (1989) Estimation of episodic and climatological skill and uncertainty in regional numerical models. Q J R Meteorol Soc 115:763-806

Brankovic C, van Maanen J (1985) The ECMWF climate system. Tech Memo 109, European Centre for MediumRange Weather Forecasts, Reading

Bromwich DH, Cullather RI, Serreze M (2000) Reanalyses depiction of the Arctic atmospheric moisture budget. In: Lewis EL (ed) The fresh water budget of the Arctic Ocean. Kluwer, Dordrecht, p 163-196

Caya D, Biner S (2004) Internal variability of RCM simulations over an annual cycle. Clim Dyn 22:33-46

Christensen JH, Christensen OB, Lopez P, van Meijgaard E, Botzet M (1996) The HIRHAM4 regional atmospheric climate model. DMI Sci Rep 96-4, Danish Meteorological Institute, Copenhagen

Christensen OB, Gaertner MA, Prego JA, Polcher J (2001) Internal variability of regional climate models. Clim Dyn $17: 875-887$

Covey C, AchutaRao KM, Cubasch U, Jones P, Lambert SJ, Mann ME, Phillips TJ, Taylor KE (2003) An overview of results from the Coupled Model Intercomparison Project. Global Planet Change 37:103-133

Cullather RI, Bromwich DH, Serreze M (2000) The atmospheric hydrological cycle over the Arctic Basin from reanalyses. Part I: comparison with observations and previous studies. J Clim 13:923-937

Dalcher A, Kalnay E (1987) Error growth and predictability in operational ECMWF forecasts. Tellus 39A:474-491

Davies HC (1976) A lateral boundary formulation for multilevel prediction models. Q J R Metorol Soc 102:405-418

Denis B, Laprise R, Caya D, Cote J (2002) Downscaling ability of one-way nested regional climate models: the bigbrother experiment. Clim Dyn 18:627-646

Dethloff K, Rinke A, Lehmann R, Christensen JH, Botzet M, Machenhauer B (1996) A regional climate model of the Arctic atmosphere. J Geophys Res 101:23401-23422

Dethloff K, Rinke A, Handorf D, Weisheimer A, Dorn W (2004) Nonlinear dynamics of the climate system. In: Fischer $H_{\text {, }}$ Kumke T, Lohmann G, Flöser G, Miller H, von Storch $H_{\text {, }}$ Negendank $\mathrm{J}$ (eds) The climate in historical times. Springer Verlag, Berlin, p 13-41

Dorn W, Dethloff K, Rinke A, Roeckner E (2003) Competition of NAO regime changes and increasing greenhouse gases and aerosols with respect to Arctic climate estimate. Clim Dyn 21:447-458

Ehrendorfer M (1997) Predicting the uncertainty of numerical weather forecasts: a review. Meteorol Z 6:147-183

Francis JA (2002) Validation of reanalysis upper-level winds in the Arctic with independent rawinsonde data. Geophys Res Lett 29:1-4

Giorgi F, Bi X (2000) A study of internal variability of a regional climate model. J Geophys Res 105:29503-29521

Lynch AH, Cullather RI (2000) An investigation of boundary forcing sensitivities in a regional climate model. J Geophys Res 105:26603-26617

Marbaix P, Gallee H, Brasseur O, van Ypersele JP (2003) Lateral boundary conditions in regional climate models: a detailed study of the relaxation procedure. Mon Weather Rev 131:461-479

Orrell D, Smith L, Barkmeijer J, Palmer TM (2001) Model error in weather forecasting. Nonlin Proc Geogr 8: $357-371$

Räisänen J (2002) $\mathrm{CO}_{2}$-induced changes in interannual tem- 
perature and precipitation variability in 19 CMIP2 experiments. J Clim 15:2395-2411

Rinke A, Dethloff K (2000) On the sensitivity of a regional Arctic climate model to initial and boundary conditions. Clim Res 14:101-113

Roeckner E, Arpe K, Bengtson L, Christoph M and 6 others (1996) The atmospheric general circulation model ECHAM4: model description and simulation of presentday climate. MPI Report No 218, Max Planck Institut, Hamburg

SEARCH (2003) SEARCH Study of Environmental Arctic Change. Implementation Strategy. Scientific Steering Committee, Polar Science Center, University of Washington, Seattle, WA

Serreze MC, Hurst CM (2000) Representation of mean Arctic precipitation from NCEP-NCAR and ERA reanalyses. J Clim 13:182-201

Serreze MC, Box JE, Barry RG, Walsh JE (1993) Characteristics of Arctic synoptic activity, 1952-1989. Meteorol Atmos Phys 51:147-164

Seth A, Giorgi F (1998) The effects of domain choice on summer precipitation simulation and sensitivity in a regional climate model. J Clim 11:2698-2712

Simmons A, Mureau R, Petroliagis T (1995) Error growth and estimates of predictability from the ECMWF forecasting

Editorial responsibility: Otto Kinne, Oldendorf/Luhe, Germany system. Q J R Meteorol Soc 121:1739-1771

Uttal T, Curry JA, McPhee MG, Perovich DK and 24 others (2002) Surface energy budget of the Arctic Ocean. Bull Am Meteorol Soc 83:255-275

von Storch H, Langenberg H, Feser F (2000) A spectral nudging technique for dynamical downscaling purposes. Mon Weather Rev 128:3664-3673

Vukicevic T, Errico RM (1990) The influence of artificial and physical factors upon predictability estimates using a complex limited-area model. Mon Weather Rev 118: 1460-1482

Walsh JE, Kattsov VM, Chapman WL, Govorkova V, Pavlova $\mathrm{T}$ (2002) Comparison of Arctic climate simulations by uncoupled and coupled global models. J Clim 15: 1429-1446

Warner TT, Peterson RA, Treadon RE (1997) A tutorial on lateral boundary conditions as a basic and potentially serious limitation to regional numerical weather prediction. Bull Am Meteorol Soc 78:2599-2617

Weisse R, Heyen H, von Storch H (2000) Sensitivity of a regional atmospheric model to a sea-state dependent roughness and the need of ensemble calculations. Mon Weather Rev 128:3631-3641

Wu W, Lynch AH, Rivers AR (2004) An approach to estimate uncertainty in a regional climate model. J Clim: in press

Submitted: April 21, 2004; Accepted: November 8, 2004 Proofs received from author(s): November 30, 2004 